\title{
Tracking bridge tilt behaviour using sensor fusion techniques
}

\author{
Karen Faulkner ${ }^{1} \cdot$ James Mark William Brownjohn ${ }^{1,2} \cdot$ Ying Wang $^{1} \cdot$ Farhad Huseynov $^{3}$
}

Received: 19 November 2019 / Revised: 23 March 2020 / Accepted: 13 April 2020 / Published online: 29 April 2020

(c) The Author(s) 2020

\begin{abstract}
The resilience of the built environment to extreme weather events is fundamental for the day-to-day operation of our transport network, with scour representing one of the biggest threats to bridges built over flowing water. Condition monitoring of the bridge using a structural health monitoring system enhances resilience by reducing the time needed to return the bridge to normal use by providing timely information on structural condition and safety. The work presented in this report discusses use of rotational measurements in structural health monitoring. Traditionally tiltmeters (which can be a form of DC accelerometer) are used to measure rotation but are known to be affected by dynamic movements, while gyroscopes react quickly to dynamic motion but drift over time. This review will introduce gyroscopes as a complementary sensor for accelerometer rotational measurements and use sensor fusion techniques to combine the measurements from both sensors to get an optimised rotational result. This method was trialled on a laboratory scaled model, before the system was installed on an in-service single-span skewed railway bridge. The rotational measurements were compared against rotation measurements obtained using a vision-based measurement system to confirm the validity of the results. An introduction to gyroscopes, field test measurement results with the sensors and their correlation with the vision-based measurement results are presented in this article.
\end{abstract}

Keywords Structural health monitoring $\cdot$ Bridges $\cdot$ Rotation $\cdot$ Gyroscopes $\cdot$ IMU $\cdot$ Sensor fusion

\section{Introduction}

The transport network in many regions around the world is vulnerable to extreme weather events. How the infrastructure responds to these hazards is crucial, not only for the protection of human life and the structures themselves, but also for socio-economic reasons. Following Storm Desmond in December 2015, 131 bridges needed urgent inspections and repairs, with the total economic damage to the region estimated to be $£ 1.3$ billion [1]. The most common cause of bridge failure in the UK and Ireland is scour, with 140 railway bridge failures during 65 separate flood events between 1846 and 2013 attributed to scour alone [2]. A review of

Karen Faulkner

kf312@exeter.ac.uk

1 Vibration Engineering Section, College of Engineering, Mathematics and Physical Sciences, University of Exeter, North Park Road, Exeter EX4 4QF, UK

2 Full Scale Dynamics Ltd., Kay Building North Park Road, Exeter EX4 4QF, UK

3 Department of Engineering, University of Cambridge, Trumpington St, Cambridge CB2 1PZ, UK
1502 bridge failures in the United States of America from 1966 to 2005, identified that $58 \%$ of these were a direct result of hydraulic action, with $32.8 \%$ and $15.5 \%$ of failures attributed to flooding and scour, respectively [3].

Scour, a dynamic phenomenon affected by a number of factors including water depth, flow speed, substructure geometry and material properties of the sediment [4], is defined as the removal of the underlying material from beneath the foundations of piers and abutments of bridges. However during periods of heavy rainfall, it is often a multihazard combination of flooding and scour which will ultimately lead to the failure of a bridge structure [5]. Numerous studies have been performed to monitor the resilience of the built environment against these multi-hazard events as a method of predicting how vulnerable the transport system is; including INFRARISK [6], INTACT [7] and RAIN [8] projects. A review of the fragility of the transport system can assist asset owners to determine the most vulnerable structures at risk on the transport network. With these studies, an understanding remains that risk cannot be removed from all bridges, which means prioritising at-risk vulnerable structures but equally means that a residual level of risk will still exist for exceptional weather events (e.g. flooding in 
2015 in Cumbria, northern England [1]) [9]. Therefore, a need exists to design a cost-effective portable system, which can be rapidly deployed and easily installed to at-risk bridge infrastructure. This DAQ logger can assess how a structure performs both during routine day-to-day environmental conditions, but that can also be used to target vulnerable but not ordinarily high-risk structures during these extreme weather events.

\subsection{Scour detection}

The ability of the built environment to withstand these extreme and multi-hazard events is crucial for the safety of the infrastructure network. There are a several scour depth monitoring techniques available $[4,10]$, but these devices can often struggle to detect evidence of scour; due to water turbidity, impact from debris to the device itself or backfill to the scour hole. Recently, scour monitoring using structural health monitoring (SHM) has been proposed as an alternative to traditional scour measurement devices. SHM offers an alternative method for estimating the integrity of a structure during periods of flooding to infer scour, though suitable analysis of in situ parameters [11]. The use of SHM reduces the reliance on visual inspections of bridges, which can often fail to find evidence of any damage to the structure, e.g. a visual inspection did not find any evidence of the visible scour at the Malahide Viaduct three days before it failed [12]. A 2001 study by the FHWA revealed that 'at least 56\% of the average Condition Ratings were incorrect with a 95\% probability from the visual inspection' [13].

There are a number of forms of SHM, with varying levels of complexity and reliability of results, ranging from anomaly detection through to damage detection [14]. A number of studies propose using changes in the dynamic properties of the structure to identify the presence of scour, where a decrease in the natural frequencies can be used to identify the presence of scour $[15,16]$. Although bridge monitoring techniques using natural frequencies of the structures have been proven to be useful for confirming the presence of scour, there is a limited number of studies in the literature on their use in actual field studies [17, 18]. Both of these studies have required testing of bridges which are known to already be undermined by scour and were used to test initially scoured and then unscoured conditions, i.e. to prove the proposed method in reverse. From an extensive literature review, there has been little evidence of a study which has managed to identify scour in unknown conditions.

Resilience can be defined as 'the capacity to recover quickly from difficulties', or from an engineering perspective, 'the ability to return to a stable steady state'. Studies have shown there is a need to integrate SHM into structures to allow for a rapid condition assessment to quantify the condition and safety of the structure following an extreme event (e.g. earthquake). Reducing time spent inspecting the structure to inform decisions on returning the structure to normal operation after an event represents improvement in resilient recovery [19]. This report will discuss a stand-alone portable SHM system which can be used to target vulnerable structures where installation of a permanent SHM system is impractical.

\subsection{Rotation-based structural health monitoring}

Rotation is an important parameter for SHM. It reflects a significant trauma to the bridge, resulting in either transient or permanent deformation, i.e. damage. Recent studies have developed various rotation-based damage detection techniques for bridge monitoring systems, including visionbased methods and measurement data from inertial sensors [20-23]. Damage detection techniques are still relatively unproven for monitoring of bridges during scour events and also require an undamaged model of the bridge. Therefore, to be able to target the most at risk bridges during a flood event, a robust stand-alone system is required; one which does not rely on previous undamaged knowledge of the structure.

This article will discuss use of rotational measurements and will propose a rotation-based condition monitoring system as an alternative SHM system for bridges. By targeting bridges most at risk during a storm event and monitoring select parameters (e.g. the quasi-static behaviour of the bridge using rotation and deflection measurements) the condition of the bridge can be readily assessed allowing for a quicker assessment. The purpose will be to monitor the structure over an extended period to establish the effects of any environmental loading on the structure.

SHM of bridges has proven to be a useful tool for engineers to assess the condition of the structure. Accelerometers and gyroscopes can both be used to obtain rotational measurements, with each sensor having their own individual strengths and weakness. Accelerometers have proven themselves as a useful tool for monitoring bridges, capable of measuring both the dynamic properties of the structure (modal properties, etc.) and the quasi-static behaviour (rotation, deflection etc.). Gyroscopes, a proven technology for positioning for other applications, have had limited use in SHM to date [24, 25].

A review of available literature has identified a lack of studies using rotational measurements for SHM purposes. Therefore, the purpose of this study is to use gyroscopes to provide more accurate rotational measurements than traditional rotation sensors allow. Section 2 of this paper will review the properties of accelerometers and their role in SHM of bridges, focussing on quasi-static behaviour. It will further introduce gyroscopes as a complimentary sensor 
which can be deployed alongside accelerometers to refine rotational measurements obtained at the structure.

Rotation data can be obtained from both accelerometers and gyroscopes. Section 3 will introduce the derivation process for rotation from both sensors and propose the use of sensor fusion techniques to obtain the best available rotation data. Sensor fusion uses the strengths of each sensor to provide an improved estimate of the true parameter value (e.g. position, rotation, etc.). The Kalman filter is a popular form of sensor fusion techniques. It applies measured values to an estimate of the system state to propose the best estimate of the state in its next iteration and will be used to demonstrate the benefits of sensor fusion.

The following sections will then introduce experiments performed to trial the methodology proposed in the earlier sections, with rotation measurements obtained using a series of accelerometers and gyroscopes. Initially, the methodology was trialled on a scaled model of a bridge in the laboratory. The set-up and results of the laboratory tests are discussed in Sects. 5 and 6, respectively. Following the successful laboratory test results, the tests were repeated on a railway bridge which forms part of a heritage railway in the UK. The test set-up and results of fieldwork performed on this bridge are discussed in Sects. 7 and 8, respectively.

\section{Introduction to inertial sensors and rotational measurements}

The traditional method for measuring rotation for SHM studies is to use tiltmeters or inclinometers [26, 27]. The concept behind an inclinometer is that it performs measurements of various responses generated by pendulum behaviour caused by gravity [26]. A number of pendulum behaviours exist, including solid mass [28], liquid [29] and gas [30, 31], and measured using resistive [32], capacitive [33], inductive [34], magnetic [35], fibre-optic [36] or optical [37] methods.

Rotation can be easily calculated in $X$ - and $Y$-directions by projecting the gravity vector on the axes of acceleration. The output of an accelerometer follows a sinusoidal pattern as it is rotated through a gravity field; therefore, acceleration can be converted into rotation using the following relationship:

$\theta=\sin ^{-1}\left(\frac{A_{x, \mathrm{out}}[g]}{1 g}\right)$,

where $\theta$ is the angle of rotation and is measured in radians. As the measured rotations are very small, the following approximation can be used:

$\sin (\theta) \cong \theta, \theta \ll 1$.
Accelerometer is a proven technology to obtain rotation measurements and is known to be stable over long measurement periods; however, they are also less sensitive to dynamic rotations of linearly accelerating structures [38] and can be affected by crosstalk from motion in other axes [25]. The advantage of gyroscopes is that they can be used where measurements of dynamic rotations are required.

\subsection{Introduction to gyroscopes}

There are two principal types of gyroscope; one which measures rotation directly and the second which measures angular velocity, from which the angle of rotation can be obtained via integration. Rotation can be measured directly with mechanical gyroscopes, which are usually very expensive. Gyroscopes which measure angular velocity are referred to as rate gyroscopes, with optical and MEMS gyroscopes the most common choice of rate gyroscope. Comprehensive introductions to gyroscopes are available $[39,40]$.

Mechanical gyroscopes, measuring angles directly, consist of a spinning wheel mounted on two gimbals, allowing friction-free movement on all three axes. As the sensor is rotated, the central spinning wheel will remain in a constant global position and the angle between the gimbals will change. The orientation of the gyroscope can then be measured by reading the angles between the gimbals. The main drawbacks to mechanical gyroscopes are that they are traditionally slow to start up and it is difficult to maintain friction free movement as the size of the sensor decreases. Gimbal lock can also be an issue, which occurs when two of the three gimbals are rotated to a parallel position and get locked together, reducing the movement of the device to two axes.

The most common forms of rate gyroscopes are optical and microelectromechanical (MEMS) gyroscopes. Optical gyroscopes are comprised of fibre-optic gyroscopes (FOG) and ring laser (RLG) gyroscopes. Both sensors work on similar principles, with the interference of light used to measure the angular velocity through the Sagnac effect. The phaseshift caused by the Sagnac effect induced by the rotation of the sensor creates a beam of light whose intensity is correlated with the angular velocity of the rotation. The accuracy of a fibre-optic gyroscope is related to the effective area of the closed optical path, which is increased by increasing the number of loops of coil within the system. The RLG sensor operates under similar principles to the FOG, also using the Sagnac effect to measure angular velocity, but mirrors are used to pass the light beam instead of a coil. Due to their accuracy, RLG is used where precision measurements are required, particularly in the aerospace industry and in inertial navigational systems. However, as the accuracy of the optical gyroscopes is dependent on the length of the path the 
light has to travel, maintaining accuracy in smaller sensors is again an issue.

MEMS gyroscopes, similar to the accelerometer equivalent, are cheaper to manufacture, and with measurement accuracies improving, are proving popular for modern inertial measurement unit (IMU) systems. As they contain no rotating parts, the sensors can be easily adapted in miniaturised form. MEMS gyroscopes are also known as vibrating structure MEMS gyroscopes and use the Coriolis effect to determine the angular velocity. The Coriolis effect states that a reference frame of mass $m$ moving with a velocity $v$ and rotating at an angular velocity $\omega$ will experience a force, $F_{c}$ :

$F_{\mathrm{c}}=-2 m(\omega \cdot v)$.

Vibratory elements within the MEMS gyroscopes are used to measure the Coriolis effect. MEMS gyroscopes consist of 2 functional parts, an actuator to generate a velocity field to produce the Coriolis effect and an accelerometer to determine the deflection in the sensing arm.

A simple example of a MEMS gyroscope consists of a device with a mass which is driven to vibrate. Recent advances in MEMS technologies have produced two popular forms of vibrating gyroscopes, with the proof mass vibrating in either a translational movement or in rotational oscillations. The frequency of the driving force is chosen to be as close to resonance as possible, to create larger amplitudes and correspondingly, larger signals. A drive loop with good amplitude control is important, as it ensures that the driven mass is moving at a constant amplitude and frequency, and, therefore, the scale factor for the sensing arms can also remain a constant.

The advantage of MEMS gyroscopes is that they can be produced in a range of sizes and can provide accurate rotational data from small components. Whilst the accuracy of the ring laser gyroscopes sets them apart, the accuracy of the MEMS devices has improved rapidly with FOG-grade capabilities now available in high-grade MEMS sensors [40]. The cost of MEMS gyroscopes is also considerably less, making them more accessible for SHM projects.

\section{Methodology}

\subsection{Sensor fusion of rotational measurements}

The position of an object is described within the $X, Y$ and $Z$-axes Cartesian co-ordinate system. Rotation of an object is defined using six degrees of freedom moving in a threedimensional body, with positive and negative movement allowed in the pitch, roll and yaw axes. Pitch and roll are used to describe rotation along the $X$ and $Y$ co-ordinate system, respectively. Yaw is used to describe rotation around the $Z$-axis co-ordinate system.

Accelerometers are used to define rotation around the horizontal axes, i.e. pitch and roll. Inclination refers to the angle of deviation from the vertical axis, with single-axis accelerometers often deployed as inclinometers. However, accelerometers cannot derive rotation about the vertical axis, i.e. yaw. To measure yaw, magnetometers are also required. However, this paper considers that for tracking the movement of a bridge under loading conditions, pitch and roll are sufficient as these are the likely consequences of scour induced deformation.

Rotation can be obtained directly from accelerometer readings, but these are known to be less sensitive to dynamic movements. Dynamic rotation can be obtained by integrating the angular velocity measurements from rate gyroscopes. However, as the measurements are cumulative, any noise in the measurements will cause the sensor to drift over time. Sensor fusion is one method which can be used to optimise both sensor measurements to utilise the best components of each sensor. Of sensor fusion techniques, the Kalman Filter is one of the predominate forms in use today.

The Kalman filter was developed by Rudolf E. Kálmán as a statistical process to produce a best estimate of the state of a system based on measured variables [41]. In other words, uncertain information about a dynamic system (i.e. the drift in time within data measured from a gyroscope) can be used to provide a reliable estimation of the correct value. The Kalman filter used by the Apollo 11 mission [42] assisted in the guidance of the spacecraft as it approached the moon. Whilst a mainstay of many industries, in particular for navigation [43-45] and autonomous vehicles [46], the Kalman filter has seen a surge in application demand as multiple sensors are increasingly adapted for everyday tasks, e.g. signal processing [47].

An introduction to the Kalman filter is presented by [48]. It can be used to track the movement or position of a structure using measured dynamic properties (e.g. rotation, acceleration, etc.). The Kalman filter provides a statistical best estimate of the next position of the system, based on the previous position, measured parameters and noise parameters of both the sensors and the system model. Without knowing the exact rotation and acceleration values at any discrete point in time, the Kalman filter recognises that at any point in time, the values for each parameter are within a certain range. Within this range of values, the filter recognises that certain values are probable than others.

The Kalman filter uses the principle that the input variables (rotation and acceleration) are random and Gaussian distributed. Each input will have an individual mean, $\mu$, and variance, $\sigma^{2}$, which represents the centre of the Gaussian random distribution (i.e. the solution that is probabilistically most likely to be correct) and the uncertainty (i.e. the error), 
respectively. A Kalman filter is a recursive estimator which uses the previous state of a linear dynamical system and measured parameters to determine the best estimate of the state at the next discrete time step. The Kalman filter works to remove the noise associated with each sensor; using inaccurate data to obtain a more accurate result.

The Kalman filter consists of two phases, prediction and update. The prediction stage consists of two elements, the predicted state estimate and the error or confidence associated with this estimate. The update stage represents the recursive element of the Kalman filter process and is used to generate the inputs for the Kalman filter at the next discrete time step.

As the Kalman filter is being used to track movement of a bridge, accelerometer and gyroscope data are sufficient to track the movement of the structure under loading. As the aim is to measure bridge tilt, i.e. rotation in the pitch and roll directions, only two DOFs are required.

\subsection{Deflection measurements}

Deflection is derived from the method presented by [49], who proposed a procedure to calculate deflection based on accelerometer and strain gauge measurements. The procedure allows for the calculation of deflection using accelerometer readings only if the bridge is instrumented with a sufficient number of inclination sensors. This procedure was used to derive deflection measurements in [50] and can be referred to for further details.

\section{Equipment}

\subsection{DAQ and sensors}

The primary purpose of the experiment was to trial a new SHM system developed by the vibration engineering section (VES) at the University of Exeter. VES has previously developed a data acquisition (DAQ) system consisting of stand-alone, time-synchronised loggers equipped with triaxial and uniaxial accelerometers. The purpose of the testing described in this article was to adapt the DAQ loggers to enable the use of uniaxial gyroscope measurements with the DAQ systems, time-synchronised with the accelerometer data.

The time synchronisation of better than $1 \mathrm{~ms}$ is achieved through $10-\mathrm{MHz}$ oven-controlled crystal oscillators (OXCOs) [51]. Each case comprises the power source, cabling and a National Instruments (NI) CompactRIO cRIO9064 to control the DAQ system. Each cRIO-9064 contains a 4-channel NI-9234 vibration input module connected to the sensors. Time synchronisation is controlled through a blank NI-9977 C-Series module, while the local timing of each logger is controlled through an NI-9402 module at $120 \mathrm{MHz}$. The DAQ loggers have proven to be a dependable monitoring system having been successfully deployed for a number of projects, including modal analysis of bridges [51] and rock-based lighthouses [52].

The DAQ loggers were developed to operate in indoor and outdoor environments, capable of maintaining synchronised data recording, better than $1 \mathrm{~ms}$, throughout the measurement period. The data are saved to an internal USB drive, but can be recovered via network file transfer at any instance during the testing process with no disruption to the acquisition process. Each DAQ logging system is protected inside an individual waterproof case, complete with an 8 amp hour internal battery. The system operates on a current draw of approximately 0.5 to $0.6 \mathrm{amps}$, allowing for $16 \mathrm{~h}$ of measurement capabilities. For longer measurement periods, the DAQ logger can be connected to a larger external battery to increase the operational capabilities of the system. The DAQ logger can be operated on mains power, though this is limited for indoor use. Solar panels were considered as an alternative power system for the logger, but were ultimately rejected to prioritise speed of installation, and as they would have no benefit when the loggers are used in an indoor environment.

However, a new DAQ system is currently in development, which will aim to extend the battery life of the logger through a larger internal battery-powered system. The new data logging system will consist of a single self-contained DAQ logger, which will contain both the larger batteries and sensors required for the Kalman filter procedure within the case itself. To maintain the IP67 rating of the new logging system [53], internal batteries will be the sole power source for the DAQ logger to eliminate any potential sources for water ingress, as it would be necessary to drill holes in the case to accommodate either external batteries or solar panels. VES has experience designing DAQ logging systems which are sealed to prevent water ingress, which requires considerable care. The priority for this system was to be robust enough to withstand the effects of extreme weather events, and, therefore, the priority for the new logging DAQ logger is for an increased internal battery power system with no external components.

The only external components of the system are the sensors and the cables to attach them to the loggers. The system is designed to be quickly mobilised and easily installed for short-term measurements on a structure, e.g. monitoring a bridge during a storm event. A complete introduction to the testing system is provided by Brownjohn et al. [51].

The system initially consisted of one master DAQ logger and three slave DAQ loggers, with each slave unit synchronised to the time clock of the master unit at the start of each test. The system has now evolved to 10 loggers, with all data 
acquisition units now operating interchangeably as either master or slave units.

The DAQ loggers were initially designed with the intention of recording acceleration measurements, with each logger configured to work with either a set of Honeywell QA-750 uniaxial accelerometers or a single Japanese Aerospace JA-70SA triaxial accelerometer. The aim of this test was to trial the DAQ loggers to operate with two Silicon Sensing CRH02-25 uniaxial gyroscopes, allowing timesynchronised measurements using both accelerometers and gyroscopes.

\subsection{Vision-based measurement system}

To test the validity of the DAQ sensor results, a vision-based Imetrum measurement system was used to determine rotation and deflection measurements. The Imetrum measuring system was developed by Imetrum Limited, UK to use image processing to track bridge movements and determine strain measurements in composite materials. The cameras are particularly useful for outdoor environments and difficult to access structures.

Deflection measurements can be obtained by positioning a target at the proposed measurement location and tracking the movement of the target as a load is applied to the structure. Similarly, rotation measurements are obtained by tracking two targets with one camera and measuring how the angle between the two targets change as the plane in which they are attached is rotated. Further information on the setup and operation of the cameras is available in [54].

Whilst the Imetrum cameras are a useful tool to verify the rotation and deflection results obtained from the sensors readings, it is not appropriate for the short-term measurements of bridges proposed by this study. Apart from allweather capability limitations, as a clear line of sight is required to each target, finding suitable locations to set up the equipment can pose a problem and maintaining visibility in poor conditions can be difficult. Therefore, the visionbased system lacks the stand-alone capabilities offered by the DAQ sensors.

\section{Laboratory testing}

To test the procedure prior to implementation in the field, an idealised scale model of a bridge was constructed in the laboratory. The bridge was constructed from steel, of length $2.5 \mathrm{~m}$ with a width of $0.2 \mathrm{~m}$, with four legs of 0.5 -m length constructed of M8 threaded bar. The set of legs at one end of the bridge was screwed to the ground to mimic a fixed support. The other end of the bridge was installed in a container of gravel, free to rotate to mimic the reduced stiffness around abutments undermined by scour (Fig. 1).

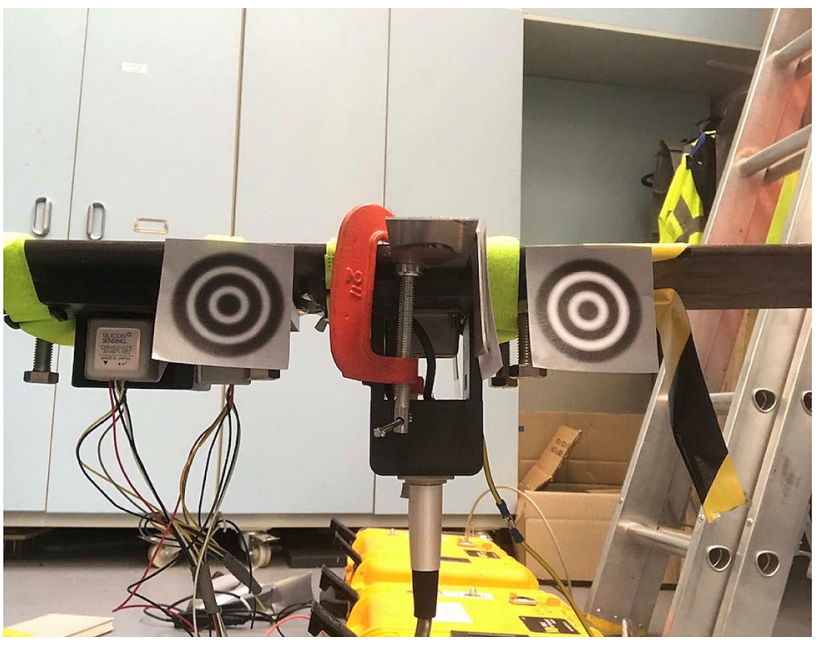

Fig. 1 The sensors, two silicon sensing CRH02-25 uniaxial gyroscopes and a Japanese Aerospace JA-70SA triaxial accelerometer, were fixed to a $3 \mathrm{D}$ printed base and attached to the bridge via magnets. The sensors were connected to the DAQ loggers in yellow plastic cases via cable. Imetrum targets were attached to the bridge to measure rotation in both pitch and roll axes

Using a weighted dumbell, traffic was simulated on the bridge by rolling the dumbell along the top of the bridge. The weights were applied twofold; (1) equal weights on either side of the dumbbell to represent traffic loading on both sides of the bridge and (2) with weight on one side of the dumbbell only, so loading would be applied to just one side of the track.

A number of simulations were performed using both unscoured and scoured conditions. Scour was applied to the right-hand side of the track, by lowering the supporting nut on the threaded bar, so the deck was free to move along the section of the threaded bar. Loading was applied equally to both sides of the bridge using a dumbbell with a $0.5-\mathrm{kg}$ weight attached to each side, totalling $1.0 \mathrm{~kg}$. Loading was applied individually to one side of the track using $2.5 \mathrm{~kg}$ or $5 \mathrm{~kg}$ attached to one side of the dumbbell.

Data were logged using two time-synchronised data loggers, one logger attached to two Silicon Sensing CRH0225 uniaxial rate gyroscopes and one logger attached to one Japanese Aerospace JA-70SA triaxial accelerometer. Simultaneous readings were recorded using the Imetrum camera system to verify the results obtained using the data loggers.

\section{Results-laboratory testing}

Time-synchronised data were recorded continuously using the DAQ loggers. Initially, a fast Fourier transform (FFT) was applied to the accelerometer data to identify the dominant frequency of the bridge, which would be used to 
determine the cut-off frequency for the low-pass filter and identified $2.6 \mathrm{~Hz}$ as the dominant frequency. Additionally, the FFT identified further peaks of $3.3 \mathrm{~Hz}$ and $3.7 \mathrm{~Hz}$ in the roll axis. An analysis of the individual 'train loadings' identified that the $3.3 \mathrm{~Hz}$ correlated with the scoured conditions and the higher frequencies correlated with the unscoured conditions.

Following the low-pass filter, rotation was calculated from the accelerometer and combined with the gyroscope data using the Kalman filter technique described in Sect. 3.1. The data were processed in MATLAB using a script created by [55], but adapted by the authors to fit the bridge and sensor set-up. Figure 2 shows pitch and roll rotation measurements obtained using scoured conditions and a $5-\mathrm{kg}$ weight on the dumbbell. The weight was used on the right-hand side of the bridge, which was the same side of the bridge that scour was added to.

Generally, there is good correlation between the results of the visual based measuring system and the sensor fusion based data acquisition system, though the maximum rotational values obtained via the sensor fusion method do not equal the maximum measured by the Imetrum system. The measurements in the roll direction show increased noise in comparison to the pitch axis, as the angles measured in the roll axis are significantly smaller than the pitch axis.

The gyroscope measuring rotation in the roll axis showed increased background noise in comparison to the gyroscope positioned in the pitch axis. This increased noise made it more difficult to isolate the true angular velocity data. However, the Kalman filter rotation angle presented for the pitch axis shows better correlation to the Imetrum data, picking up the dynamics of the beam bridge better than the accelerometer data in isolation.

\section{Field testing}

The aim of the test was to determine the rotation and deflection measurements of a railway bridge under traffic loading. The bridge tested is a historic railway bridge operated by the West Somerset Railway (WSR). The bridge is a 14.8-m steel girder single-span bridge, with two masonry abutments and carrying a single track (Fig. 3). The abutments are orientated with a skew angle of $60^{\circ}$ to the main beams of the bridge. The abutments consist of the main wall and two integrated wing walls. The height of the main and wing walls above the ground surface is approximately $5.0 \mathrm{~m}$ and $3.8 \mathrm{~m}$, respectively. The bridge was originally constructed in the $1870 \mathrm{~s}$ of wrought iron plate girders, carrying a timber deck supporting the ballasted track bed, with the abutments constructed from local stone. A comprehensive maintenance and repair programme to the bridge was carried out in 2011 with the deck replaced by steel beams.

Steam locomotives primarily operate on the route, with a number of diesel multiple unit (DMU) trains also
Fig. 2 Rotation calculated using in the (i) roll and (ii) pitch axes using the $5-\mathrm{kg}$ plate on the scoured side of the model bridge
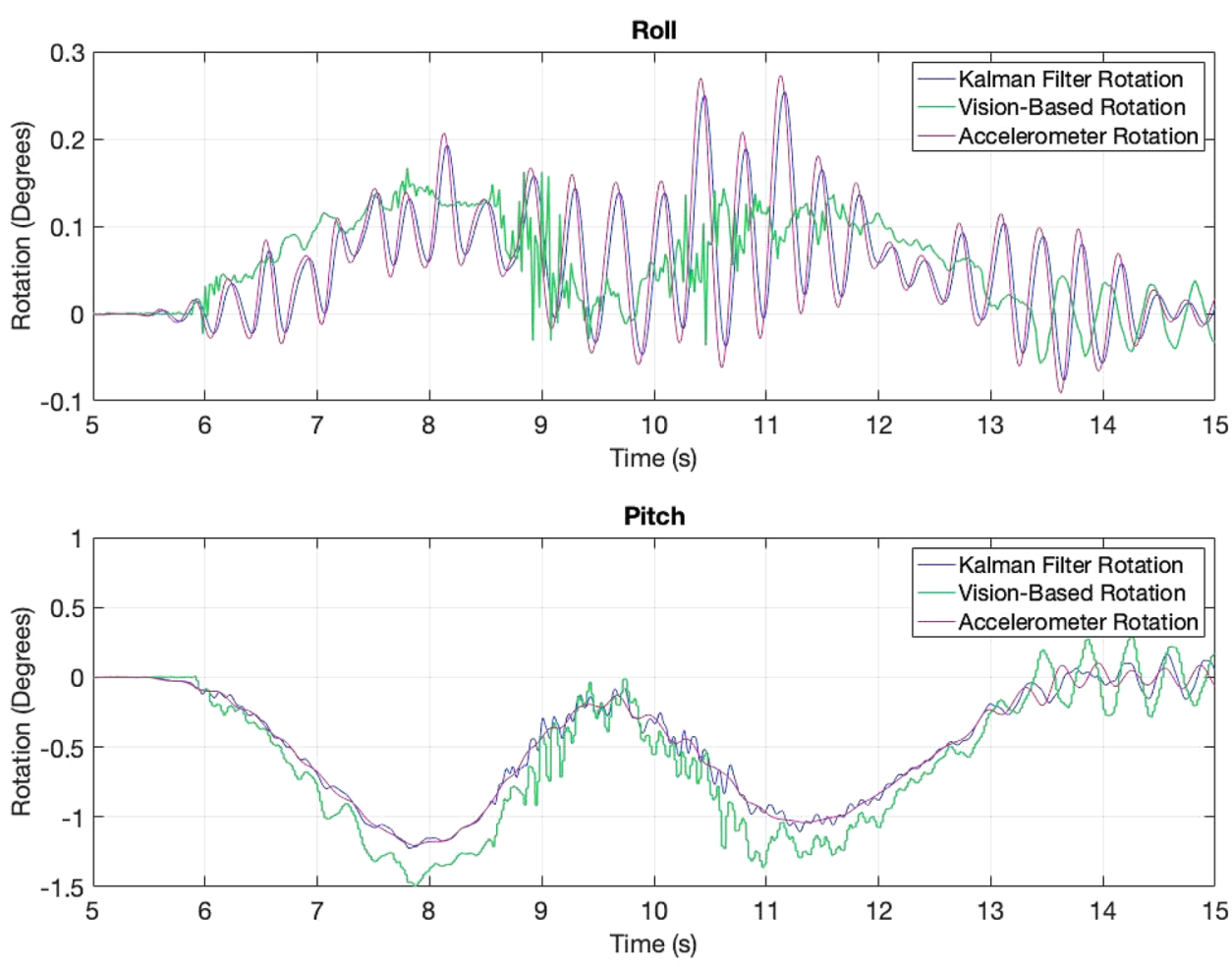


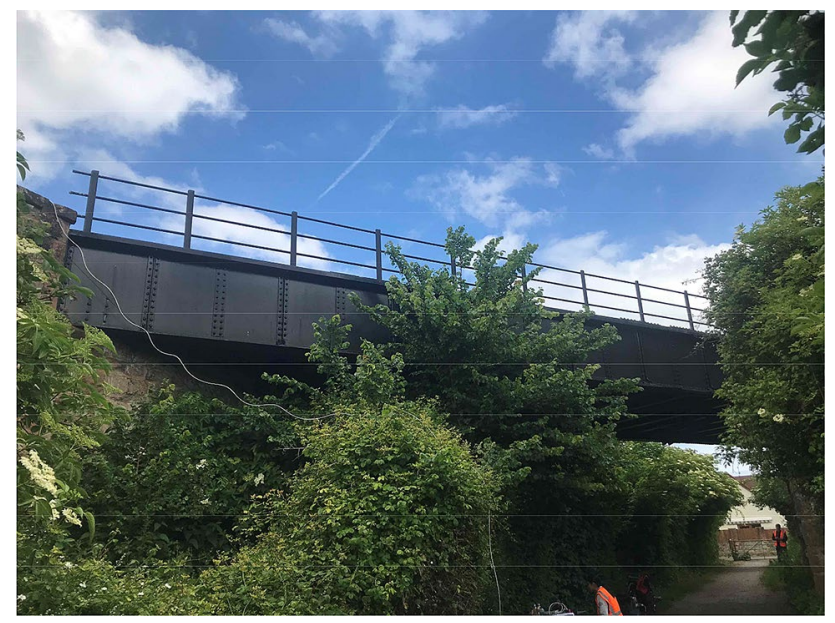

Fig. 3 Mineral Line Bridge, West Somerset Railway. A steel girder bridge skewed at an angle of $60^{\circ}$ to the stone abutments beneath

in operation. The steam locomotives have a higher axial load than their diesel counterparts. Due to their increased popularity with passengers, they also impart a greater live load in the following carriages than what is evident for the identical carriages following the diesel locomotives [50]. Three steam trains were in operation during testing, described in in Table 1. The Bradley Manor and 7F Class 2-8-0 53,808 locomotives operate as locomotive plus tender, whereas the Steam Engine 7752 operates independent of a tender.

Previous testing at the site confirmed that significant rotations are created on the bridge when the bridge is subject to loading from passing trains [50]. Therefore, the objective of this testing was twofold; primarily to perform an academic study to measure deck rotations in the pitch and roll direction using the sensor fusion technique developed by VES and secondary to provide deck rotations and deflections under traffic loading to the bridge owner.

\subsection{Rotational measurements}

Sensors were installed along the southern side of the bridge deck to obtain angular velocity and acceleration measurements under ambient loading from passing trains. The DAQ system, described in Sect. 4.1, is easily transportable and is housed within a protective plastic case, which is designed to be crushproof, waterproof and dustproof. Additionally, because of the casing, it is ideal for operation in outdoor environments, with only the sensor and the wire connector exposed to the weather conditions.

Two Silicon Sensing CRH02-25 uniaxial rate gyroscopes and a single Japanese Aerospace JA-70SA triaxial accelerometer were installed at the quarter-span location to combine together to obtain rotational measurements using the Kalman filter approach. In addition, two Honeywell QA-750 uniaxial accelerometers were installed in the $X$ - and $Y$-axes at each abutment, mid-span and three-quarter-span locations, to obtain rotation and deflection measurements.

The vision-based measurement system consisted of two targets positioned at quarter-span on the deck, with two cameras positioned to the north of the bridge. The complete test set-up is presented in Fig. 4.

\section{Results-field testing}

The DAQ loggers and sensors recorded data continuously throughout the entire monitoring period. The vision-based logging system recorded data over the period immediately prior, during and after each train has passed.

As per the laboratory testing, an FFT of the complete dataset was performed to identify the dominant frequency evident in the structure to determine the cut-off frequency for the low-pass filter. The Kalman filter was applied using the process described in Sects. 3.1 and 6. A peak of $0.3 \mathrm{~Hz}$ was identified in the gyroscope results, with no clear peak

Table 1 Timetable of trains during the test period

\begin{tabular}{lllllccc}
\hline Time & Locomotive & $\begin{array}{l}\text { Locomotive } \\
\text { configuration }\end{array}$ & $\begin{array}{l}\text { Number of } \\
\text { carriages }\end{array}$ & Year built & Length (m) & $\begin{array}{l}\text { Gross weight loco- } \\
\text { motive (tonnes) }\end{array}$ & $\begin{array}{l}\text { Gross weight } \\
\text { tender } \\
\text { (tonnes) }\end{array}$ \\
\hline 10:40 & Steam Engine 7752 & Locomotive & 6 & 1930 & 9.5 & 47.5 & $\mathrm{n} / \mathrm{a}$ \\
$11: 10$ & Bradley Manor & Locomotive & 7 & 1938 & 18.8 & 69.8 & 40.0 \\
$12: 20$ & 7F Class 2-8-0 53,808 & Tender & 7 & 1925 & 17.9 & 65.8 & 43.4 \\
$13: 10$ & Bradley Manor & Tender & 7 & 1938 & 18.8 & 69.8 & 40.0 \\
$13: 30$ & Steam Engine 7752 & Locomotive & 6 & 1930 & 9.5 & 47.5 & $\mathrm{n} / \mathrm{a}$ \\
$15: 05$ & 7F Class 2-8-0 53,808 & Tender & 7 & 1925 & 17.9 & 65.8 & 43.4 \\
15:36 & Bradley Manor & Locomotive & 7 & 1938 & 18.8 & 69.8 & 40.0 \\
$16: 14$ & Steam Engine 7752 & Locomotive & 6 & 1930 & 9.5 & 47.5 & $\mathrm{n} / \mathrm{a}$ \\
\hline
\end{tabular}

${ }^{\mathrm{a}}$ Locomotive in reverse 


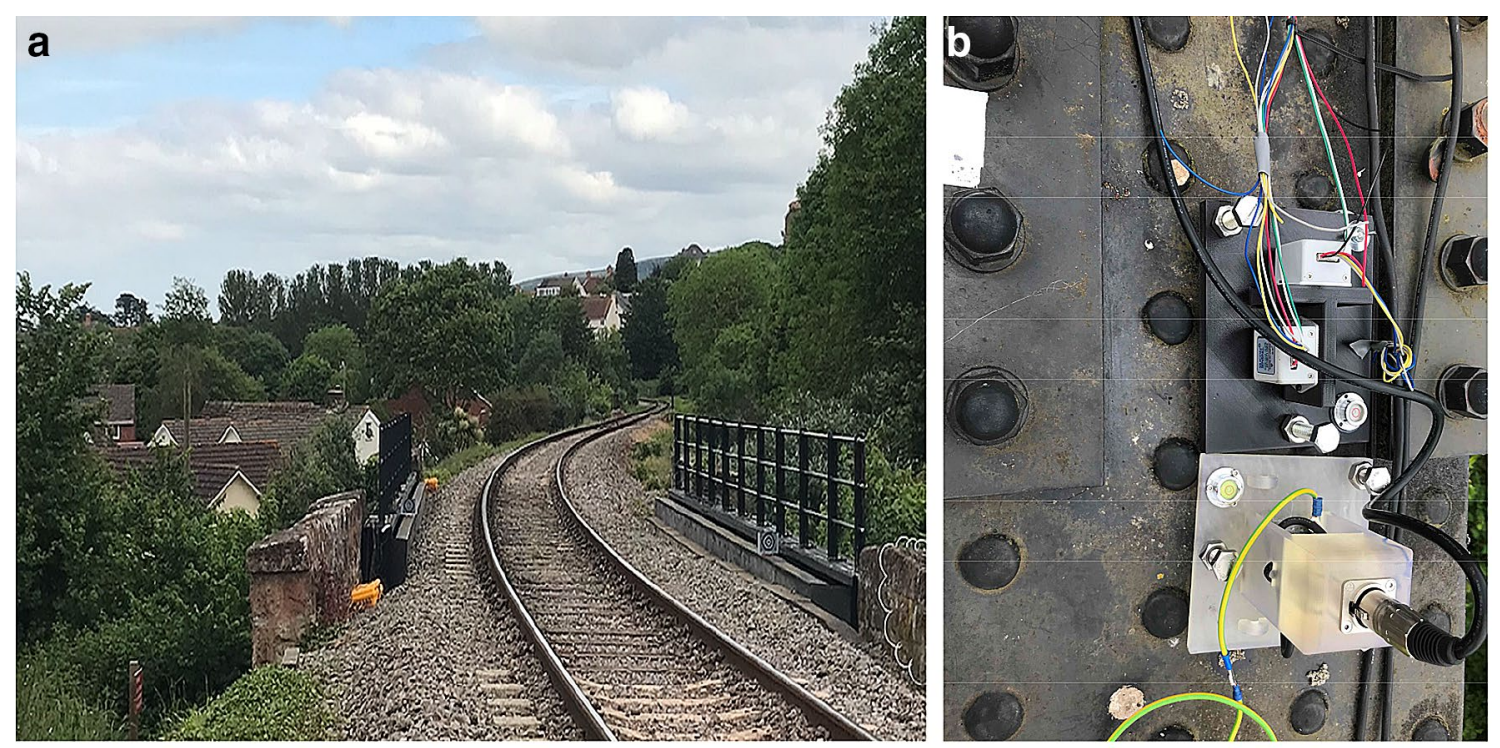

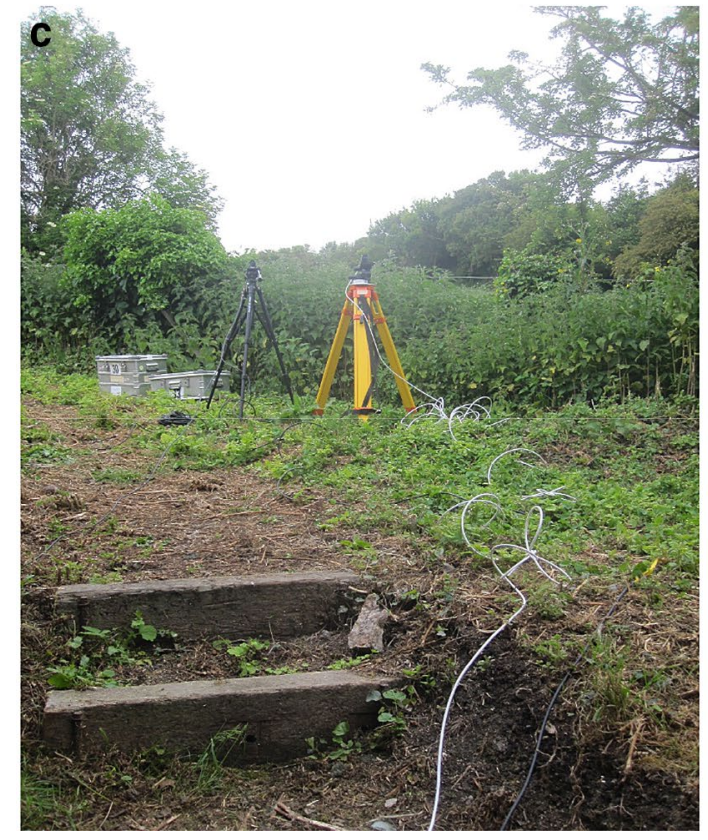

Fig. 4 a Set-up of the sensors and DAQ loggers on the South side of the track, $\mathbf{b}$ quarter-span set-up consisting of two Silicon Sensing CRH02-25 uniaxial gyroscopes and one Japanese Aerospace JA-

evident in the accelerometer results. A low-pass filter of $4.0 \mathrm{~Hz}$ was applied to the accelerometer data to remove noisy data.

Rotation was calculated from the gyroscope and accelerometer data using the method described in Sect. 3.1. Rotation was measured using the vision-based system through the two targets positioned at quarter-span of the bridge. The rotation results for Train 8 using both testing methods are presented in Fig. 5.

The calculated rotation results for the carriages derived from the Kalman Filter process show good correlation with

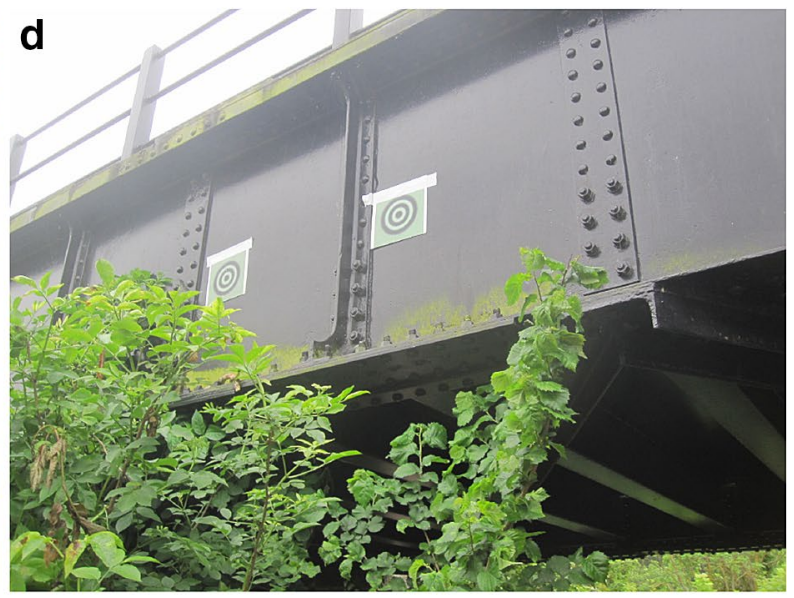

70SA triaxial accelerometer, c Imetrum Camera measuring system positioned to the South of the bridge and $\mathbf{d}$ two Imetrum targets positioned at quarter-span location to measure rotation and deflection

the measured parameters from the Imetrum camera. However, the rotation derived by the sensors cannot match the peak value for the locomotive achieved by the vision-based measurements. The difference between the vision-based measurements and those based on the sensor fusion is attributed to positioning error laying out the sensors and targets at the quarter-span. The sensors were positioned directly at the quarter-span location on the bridge deck, but the mid-point between the two vision-based targets was positioned as close as was easily accessible to the quarter-span point. 
Fig. 5 Rotation calculated at quarter-span during Train 8, with rotation from the Kalman Filter described above, the Imetrum camera system and calculated from accelerometer readings only

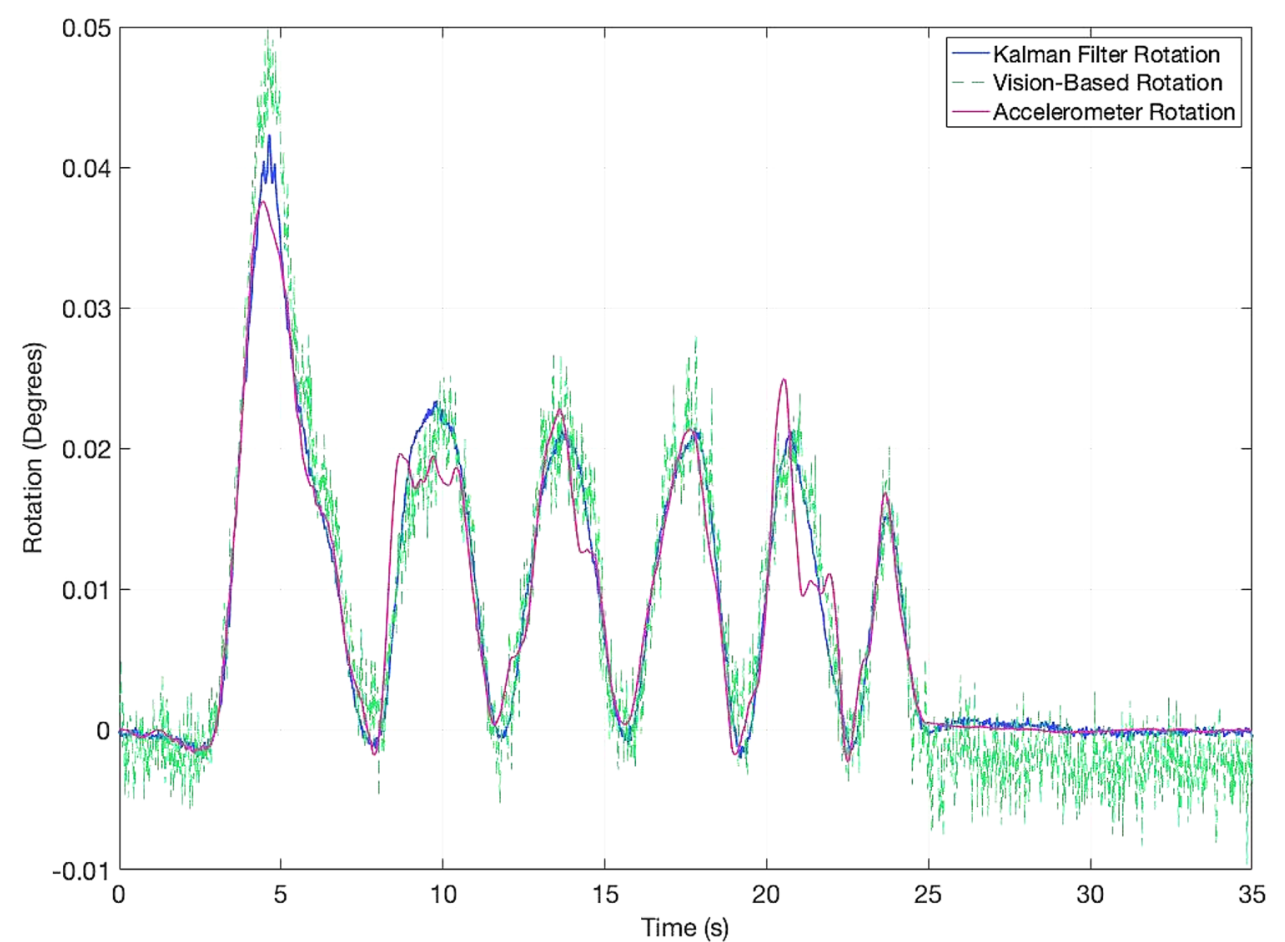

To judge whether the addition of the gyroscope adds meaningful data to the SHM approach, a comparison of rotation determined from accelerometer alone versus rotation derived from the sensor fusion technique is made. Figure 5 shows the sensor fusion rotation correlates better with the Imetrum data in comparison to the rotation calculated from the accelerometer only data. This means that whilst the SHM approach does not achieve an exact match for the Imetrum results, the addition of the gyroscope data to the accelerometer data reduces the error between the accelerometer only data and the Imetrum data, i.e. is closer to the true rotation experienced by the bridge.

The rotation obtained from the accelerometer readings over-estimated the rotation for a number of the carriages which followed the leading locomotive, but that the rotation from the sensor fusion method showed better correlation to the Imetrum readings for these carriages. This shows that the rotation from the Kalman filter not only provides a better measurement of the maximum rotation of the leading locomotive, but also can be used to provide improved rotational measurements for the carriages as well which were amplified by the accelerometer only data.

Figure 6 below plots the deflection measurements obtained fusing the Imetrum camera against the deflection determined from the rotation calculated from the sensor fusion technique, described in Sect. 3.1, with the deflection calculations derived by Helmi et al. [49].

Deflection was calculated from sensor measurements to prove the validity of the rotation measurements derived from the sensor fusion techniques. There is good correlation between the deflection derived through sensor fusion techniques and the measured Imetrum deflection data, which confirms that the sensor fusion technique offers a viable alternative quasi-static measurement system. In reverse of the trend displayed with the rotational measurements in Fig. 5, the Imetrum values are less than the inferred deflection results. As the results from both methods are in phase, the source of the discrepancy between the methods is attributed to the integration process in the calculation of the deflection measurements (Table 2).

\section{Future work}

The method discussed in this paper shows rotational measurements achieved using fusion of accelerometer and gyroscope data using the Kalman filter, presented as rotation (in the pitch axis) as trains passed over the bridge. During the data acquisition phase, sensors were placed on the bridge to measure rotation in the roll axis, but it was not possible to confirm the results of the sensor fusion with the vision-based measurement system. As the targets were positioned on either side of the deck, it was necessary to use two cameras since a clear line of sight to both cameras could not be maintained when the train was passing along the bridge. Therefore, rotation could not be measured directly and had to be inferred from displacement 
Fig. 6 Deflection readings at quarter-span calculated from the sensor fusion technique to determine rotation, using the deflection calculation result presented by Helmi et al. [49], and measured Imetrum deflection results

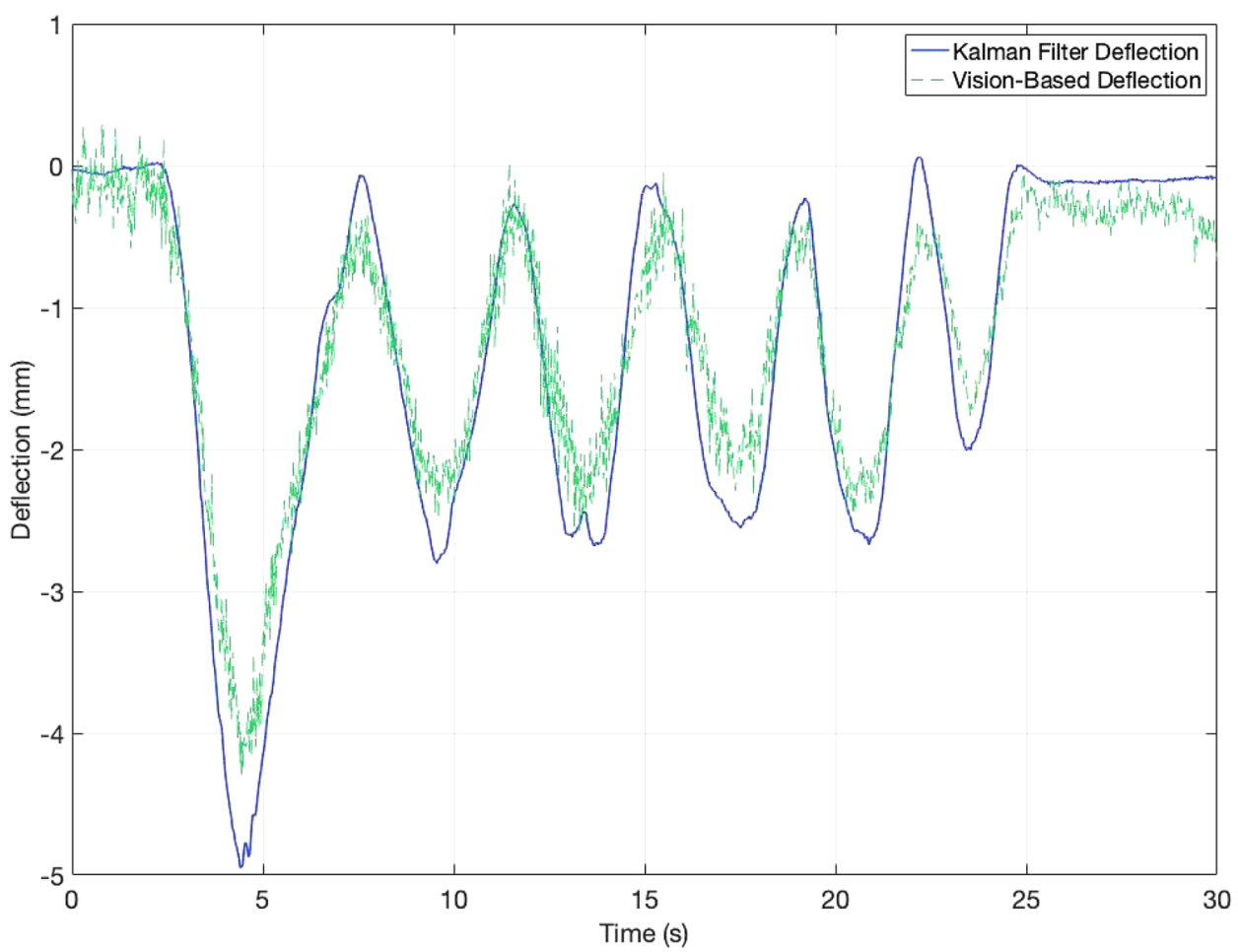

Table 2 Summary of maximum rotations and deflections at quarterspan of the bridge, obtained using gyroscope and accelerometer data with the Kalman filter

\begin{tabular}{llll}
\hline Train & Locomotive & $\begin{array}{l}\text { Maximum } \\
\text { rotation }\left(^{\circ}\right)\end{array}$ & $\begin{array}{l}\text { Maximum } \\
\text { deflection } \\
(\mathrm{mm})\end{array}$ \\
\hline 1 & Steam Engine 7752 & 0.046 & 4.77 \\
2 & Bradley Manor & 0.043 & 5.87 \\
3 & 7F Class 2-8-0 53,808 & 0.037 & 5.49 \\
4 & Bradley Manor & 0.041 & 5.58 \\
5 & Steam Engine 7752 & 0.037 & 4.81 \\
6 & 7F Class 2-8-0 53,808 & 0.040 & 5.95 \\
7 & Bradley Manor & 0.042 & 5.33 \\
8 & Steam Engine 7752 & 0.042 & 4.95 \\
\hline
\end{tabular}

measurements. Coupled with the skewed nature of the bridge, it was difficult to obtain clear roll rotational measurements. Therefore, the aim for the next bridge test is to capture the higher rate rotation measurements, as shown in the laboratory test results.

\section{Conclusion}

Scour is the most common cause of bridge failure and bridges remain vulnerable to the threat of partial or complete failure in periods of extreme weather, particularly flooding. SHM systems offer a credible alternative to traditional scour monitoring devices, which can give an indication of the presence of scour but do not inform the bridge owners about the condition of the bridge. This paper presents a SHM system which uses rotational measurements to define the quasi-static behaviour of a bridge under traffic loading.

This paper has described the application of a rotational measurement system, which operates by measuring quasistatic and dynamic measurements of the bridge using accelerometers and gyroscopes. Through sensor fusion using the Kalman filter, an improved rotational measurement can be obtained.

The method was trialled experimentally on a singlespan skewed railway bridge under traffic loading and compared against rotation measurements obtained from a reference vision-based measurement system. The data obtained from the sensor fusion techniques compared favourably against the reference system results and offered better correlation that the rotation results obtained using accelerometer data only.

The results performed in the laboratory offered good correlation in both the pitch and roll axes, but measurements in the roll axis were not obtained during the field test. In future, the test will be repeated to prove the application.

Acknowledgements The first author gratefully acknowledges the financial support of the UK Engineering and Physical Sciences Research Council (EPSRC) through a research studentship. The initial 
development of the DAQ logging system was performed using funding by the Engineering and Physical Sciences Research Council (Grant EP/ N017897/1 and EP/ N017803). The Vibration Engineering Section at the University of Exeter would like to thank the West Somerset Railway for their continued co-operation and support in allowing access to the railway infrastructure and assistance from employees and volunteers.

Open Access This article is licensed under a Creative Commons Attribution 4.0 International License, which permits use, sharing, adaptation, distribution and reproduction in any medium or format, as long as you give appropriate credit to the original author(s) and the source, provide a link to the Creative Commons licence, and indicate if changes were made. The images or other third party material in this article are included in the article's Creative Commons licence, unless indicated otherwise in a credit line to the material. If material is not included in the article's Creative Commons licence and your intended use is not permitted by statutory regulation or exceeds the permitted use, you will need to obtain permission directly from the copyright holder. To view a copy of this licence, visit http://creativecommons.org/licenses/by/4.0/.

\section{References}

1. Lamb R, Szönyi M, May P (2015) Flooding after storm desmond. Zurich Insurance Group Ltd, Zurich

2. van Leeuwen Z, Lamb R (2014) Flood and scour related failure incidents at railway assets between 1846 and 2013. Project W134224. JBA Trust 30:20

3. Zheng W (2013) FHWA/MS-DOT-RD-13-229: instrumentation and computational modeling for evaluation of bridge substructures across waterways

4. Deng L, Cai CS (2010) Bridge scour: prediction, modeling, monitoring, and countermeasures-review. Pract Period Struct Des Constr 15:125-134. https://doi.org/10.1061/(ASCE)SC.19435576.0000041

5. Wardhana K, Hadipriono FC (2003) Analysis of recent bridge failures in the United States. J Perform Constr Facil 17:144-150. https://doi.org/10.1061/(ASCE)0887-3828(2003)17:3(144)

6. Clarke J, Obrien E (2016) A multi-hazard risk assessment methodology, stress test framework and decision support tool for transport infrastructure networks. Transp Res Proced 14:1355-1363. https://doi.org/10.1016/j.trpro.2016.05.208

7. Tagg A, Raïkkönen M, Mäki K, Collell MR (2016) Impact of extreme weather on critical infrastructure: the EU-INTACT risk framework. E3S Web Conf. https://doi.org/10.1051/e3sco nf/20160707007

8. Nogal M, O'Connor A, Caulfield B, Brazil W (2016) A multidisciplinary approach for risk analysis of infrastructure networks in response to extreme weather. Transp Res Proced 14:78-85. https ://doi.org/10.1016/j.trpro.2016.05.043

9. Lamb R, Garside P, Pant R, Hall JW (2019) A probabilistic model of the economic risk to Britain's railway network from bridge scour during floods. Risk Anal. https://doi.org/10.1111/risa.13370

10. Prendergast LJ, Gavin K (2014) A review of bridge scour monitoring techniques. J Rock Mech Geotech Eng 6:138-149

11. Abdulkarem M, Samsudin K, Rokhani FZ, Rasid MFA (2019) Wireless sensor network for structural health monitoring: a contemporary review of technologies, challenges, and future direction. Struct Health Monit. https://doi.org/10.1177/1475921719 854528

12. Benn $\mathbf{J}$ (2013) Railway bridge failure during flooding in the UK and Ireland. Proc Inst Civ Eng Forensic Eng 166:163-170. https ://doi.org/10.1680/feng.2013.166.4.163
13. Pines D, Aktan AE (2002) Status of structural health monitoring of long-span bridges in the United States. Prog Struct Eng Mater 4:372-380. https://doi.org/10.1002/pse.129

14. Webb GT, Vardanega PJ, Middleton CR (2014) Categories of SHM deployments: technologies and capabilities. J Bridg Eng 20:20

15. Prendergast LJ, Hester D, Gavin K (2016) Determining the presence of scour around bridge foundations using vehicle-induced vibrations. J Bridg Eng. https://doi.org/10.1061/(ASCE)BE.19435592.0000931

16. Elsaid A, Seracino R (2014) Rapid assessment of foundation scour using the dynamic features of bridge superstructure. Constr Build Mater 50:42-49

17. Kariyawasam K, Fidler P, Talbot J, Middleton C (2019) Field deployment of an ambient vibration-based scour monitoring system at Baildon Bridge, UK. In: International conference on smart infrastructure and construction 2019 (ICSIC), pp 711-719

18. Foti S, Sabia D (2010) Influence of foundation scour on the dynamic response of an existing bridge. J Bridg Eng 16:295-304. https://doi.org/10.1061/(Asce)Be.1943-5592.0000146

19. Zhang Y, Ayyub B, Huang H (2018) Enhancing civil infrastructure resilience with structural health monitoring. Resil Eng Urban Tunnels 20:3-12

20. Huseynov F, Kim C, OBrien EJ et al (2020) Bridge damage detection using rotation measurements-experimental validation. Mech Syst Signal Process 135:106380. https://doi. org/10.1016/j.ymssp.2019.106380

21. Sung SH, Lee JH, Park JW et al (2014) Feasibility study on an angular velocity-based damage detection. Meas Sci Technol 25:75009. https://doi.org/10.1088/0957-0233/25/7/075009

22. Alten K, Ralbovsky M, Vorwagner A et al (2017) Evaluation of different monitoring techniques during damage infliction on structures. Proced Eng 199:1840-1845. https://doi. org/10.1016/j.proeng.2017.09.106

23. Jalinoos F, Amjadian M, Agrawal AK et al (2020) Experimental evaluation of unmanned aerial system for measuring bridge movement. J Bridg Eng 25:1-12. https://doi.org/10.1061/ (ASCE)BE.1943-5592.0001508

24. Liao Y, Kiremidjian AS, Rajagopal R, Loh C-H (2016) Angular velocity-based structural damage detection. In: Proceedings of the sensors and smart structures technologies for civil, mechanical, and aerospace systems conference, Las Vegas, Nevada, United States

25. Liu C, Park JW, Spencer BF et al (2017) Sensor fusion for structural tilt estimation using an acceleration-based tilt sensor and a gyroscope. Smart Mater Struct. https://doi.org/10.1088/1361$665 \mathrm{X} / \mathrm{aa} 84 \mathrm{a} 0$

26. Ha D, Park H, Choi S, Kim Y (2013) A wireless MEMS-based inclinometer sensor node for structural health monitoring. Sensors 13:16090-16104. https://doi.org/10.3390/s131216090

27. Shenton III H, Fernandez M, Ramanna N, et al (2015) Structural health monitoring of a cable-stayed bridge: using tiltmeter data to determine edge girder deflections. SHMII 2015-7th international conference on structural health monitoring of intelligent infrastructure

28. Chang CI, Tsai MH, Liu YC, et al (2011) Design and implementation of an extremely large proof-mass CMOS-MEMS capacitive tilt sensor for sensitivity and resolution improvement. In: 2011 16th international solid-state sensors, actuators and microsystems conference TRANSDUCERS'11, pp 1104-1107. https://doi.org/10.1109/TRANSDUCERS.2011.5969190

29. Liu S, Zhu R (2017) Micromachined fluid inertial sensors. Sensors 17:367. https://doi.org/10.3390/s17020367

30. Crescini D, Romani M (2011) Thick-film inclinometer based on free convective motion of an 
heating air mass. Lect Notes Electr Eng 91:367-371. https:// doi.org/10.1007/978-94-007-1324-6_58

31. Zhang F (2004) The accelerometer and tilt sensor based on natural convection gas pendulum. In: International conference on information acquisition. IEEE, pp 122-125

32. Zhang W, Zhu H, Lee JE-Y (2015) Piezoresistive transduction in a double-ended tuning fork SOI MEMS resonator for enhanced linear electrical performance. IEEE Trans Electron Devices 62:1596-1602. https://doi.org/10.1109/TED.2015.2414272

33. Zhao L, Yeatman EM (2007) Micro capacitive tilt sensor for human body movement detection. IFMBE Proc 13:195-200

34. Olaru R, Dragoi DD (2005) Inductive tilt sensor with magnets and magnetic fluid. Sens Actuators A Phys 120:424-428. https://doi. org/10.1016/j.sna.2005.01.015

35. Olaru R, Cotae C (1997) Tilt sensor with magnetic liquid. Sens Actuators A Phys 59:133-135. https://doi.org/10.1016/S0924 $-4247(97) 80162-8$

36. Antunes PFC, Marques CA, Varum H, Andrél PS (2012) Biaxial optical accelerometer and high-angle inclinometer with temperature and cross-axis insensitivity. IEEE Sens J 12:2399-2406. https ://doi.org/10.1109/JSEN.2012.2190763

37. Frazão O, Falate R, Fabris JL et al (2006) Optical inclinometer based on a single long-period fiber grating combined with a fused taper. Opt Lett 31:2960. https://doi.org/10.1364/ol.31.002960

38. Brownjohn J, Rizos C, Tan G-H, Pan T-C (2004) Real-time longterm monitoring of static and dynamic displacements of an office tower, combining RTK GPS and accelerometer data. In: 1st FIG international symposium on engineering surveys for construction works and structural engineering Nottingham, United Kingdom, 28 June-1 July 2004

39. Woodman OJ (2007) An introduction to inertial navigation

40. Passaro VMN, Cuccovillo A, Vaiani L et al (2017) Gyroscope technology and applications: a review in the industrial perspective. Sens (Switzerland). https://doi.org/10.3390/s17102284

41. Kalman RE (1960) A new approach to linear filtering and prediction problems. J Basic Eng 82:35-45

42. Grewal M, Andrews A (2010) Applications of Kalman filtering to aerospace: 1960 to present. IEEE Control Syst Mag 30:69-78

43. Barczyk M, Lynch AF (2013) Invariant observer design for a helicopter UAV aided inertial navigation system. IEEE Trans Control Syst Technol 21:791-806. https://doi.org/10.1109/ TCST.2012.2195495

44. Lim CH, Lim TS, Koo VC (2012) Design and development of a real-time GPS-aided SINU system. Int J Adv Robot Syst 9:1-9. https://doi.org/10.5772/52681
45. Xincun Y, Yongzhong O, Fuping S, Hui F (2013) Kalman filter applied in underwater integrated navigation system. Geod Geodyn 4:46-50. https://doi.org/10.3724/sp.j.1246.2013.01046

46. Garcia-Bedoya O, Ferreira JV (2018) Sensor fusion tests for an autonomous vehicle, using Extended Kalman Filter. J Eng Sci Technol Rev 11:1-8. https://doi.org/10.25103/jestr.113.01

47. Auger F, Hilairet M, Guerrero JM et al (2013) Industrial applications of the kalman filter: a review. IEEE Trans Ind Electron 60:5458-5471. https://doi.org/10.1109/TIE.2012.2236994

48. Kim Y, Bang H (2018) Introduction to Kalman filter and its applications. Kalman filter. IntechOpen, Rejika

49. Helmi K, Taylor T, Zarafshan A, Ansari F (2015) Reference free method for real time monitoring of bridge deflections. Eng Struct 103:116-124. https://doi.org/10.1016/j.engstruct.2015.09.002

50. Faulkner K, Huseynov F, Brownjohn J, Xu Y (2018) Deformation monitoring of a simply supported railway bridge under varying dynamic loads. In: Maintenance, safety, risk, management and life-cycle performance of bridges: proceedings of the ninth international conference on bridge maintenance, safety and management (IABMAS 2018), 9-13 July 2018, Melbourne, Australia. pp 1484-1491

51. Brownjohn JMW, Au S-K, Zhu Y et al (2018) Bayesian operational modal analysis of Jiangyin Yangtze River Bridge. Mech Syst Signal Process 110:210-230. https://doi.org/10.1016/j.ymssp .2018.03.027

52. Brownjohn JMW, Raby A, Bassitt J et al (2018) Experimental modal analysis of British rock lighthouses. Mar Struct 62:1-22. https://doi.org/10.1016/j.marstruc.2018.07.001

53. International Electrotechnical Commission (2013) Degrees of protection provided by enclosures (IP Code)(IEC 60529: 1989+ A1: 1999+ A2: 2013)

54. Xu Y, Brownjohn JMW, Hester D, Koo KY (2017) Long-span bridges: enhanced data fusion of GPS displacement and deck accelerations. Eng Struct 147:639-651. https://doi.org/10.1016/j. engstruct.2017.06.018

55. Salmony P IMU attitude estimation. In: https://philsal.co.uk. https ://philsal.co.uk/projects/imu-attitude-estimation. Accessed 01 Mar 2019. Accessed 1 Mar 2019

Publisher's Note Springer Nature remains neutral with regard to jurisdictional claims in published maps and institutional affiliations. 